\title{
Construct development: The Suicide Trigger Scale (STS-2), a measure of a hypothesized suicide trigger state
}

\author{
Zimri Yaseen ${ }^{1 *}$, Curren Katz ${ }^{1}$, Matthew S Johnson², Daniel Eisenberg ${ }^{3}$, Lisa J Cohen ${ }^{1}$, Igor I Galynker ${ }^{1}$
}

\begin{abstract}
Background: This study aims to develop the construct of a 'suicide trigger state' by exploring data gathered with a novel psychometric self-report instrument, the STS-2.

Methods: The STS-2, was administered to 141 adult psychiatric patients with suicidal ideation. Multiple statistical methods were used to explore construct validity and structure.

Results: Cronbach's alpha (0.949) demonstrated excellent internal consistency. Factor analyses yielded twocomponent solutions with good agreement. The first component described near-psychotic somatization and ruminative flooding, while the second described frantic hopelessness. ROC analysis determined an optimal cut score for a history of suicide attempt, with significance of $p<0.03$. Logistic regression analysis found items sensitive to history of suicide attempt described ruminative flooding, doom, hopelessness, entrapment and dread.

Conclusions: The STS-2 appears to measure a distinct and novel clinical entity, which we speculatively term the 'suicide trigger state.' High scores on the STS-2 associate with reported history of past suicide attempt.
\end{abstract}

\section{Background}

Though many chronic factors placing an individual at increased risk for suicide are well established, the acute factors that lead a person to make a suicide attempt (SA) are not known. Chronic risk factors include suicidal ideation (SI), history of suicide attempts, severe psychopathology, history of psychiatric hospitalization, substance abuse, and poor social supports [1,2]. Among these, SI and history of previous SA are most prominent and most relied upon in general clinical practice [3-7].

At present, however, no instruments are well established for the prediction of imminent SA [7]. Moreover, current measures of suicidality, including the Suicide Assessment Scale,[8-10] Suicide Intent Scale, [11,12] and Motto and Bostrom's proposed scale, [13] rely heavily on self-report of overt suicidal thoughts and plans. However, acutely suicidal individuals often deny or hide their suicidal intent, $[14,15]$ and the presence of a plan for suicide is a poor predictor of attempt, as many

\footnotetext{
* Correspondence: zsyaseen@gmail.com

${ }^{1}$ Beth Israel Medical Center, New York, New York, USA

Full list of author information is available at the end of the article
}

attempters report only fleeting ideation and no premeditated plan [4]. In fact, a recent study reported an average interval of only 10 minutes between the onset of SI and the actual suicidal act [16].

Past research suggests that transition from SI to SA may be triggered by specific affective, behavioral, and cognitive factors [17-19]. However, the exact nature of these "trigger" factors or whether they constitute a distinct "trigger state" is not known. Esposito et al., [17] reported that in adolescents, after controlling for depression, only anger and affect dysregulation differentiated multiple from single suicide attempters. Nock and Kazdin [18] have identified negative automatic thinking as a risk factor for suicide attempts. This type of cognition might be related to the "diffuse ruminative thought process" [20] characteristic of psychosis. Indeed, Radomsky et al., [21] showed that $30.2 \%$ of patients with psychosis make a suicidal attempt at some point in their life.

Furthermore, although controversial, a growing body of evidence links panic attacks to suicidal behavior in patients with depression $[22,23]$. It has been reported that this link persists even when controlling for depression, substance abuse and sociodemographic characteristics $[22,23]$.
C Biomed Central

C 2010 Yaseen et al; licensee BioMed Central Ltd. This is an Open Access article distributed under the terms of the Creative Commons Attribution License (http://creativecommons.org/licenses/by/2.0), which permits unrestricted use, distribution, and reproduction in any medium, provided the original work is properly cited. 
Weissman et al.,[24] found that $20 \%$ of subjects with panic disorder and $12 \%$ of those with panic attacks had made suicide attempts.

Finally, Schnyder et al., [25] observed that panic and self-report of "loss of control" seems to be a distinct state that occurs before individuals attempt suicide, while Busch et al., [15] found in an acute psychological autopsy study of 76 completed inpatient suicides, that nearly 80 percent both denied suicidal ideation in the days before their suicides and, using items from the Schedule for Affective Disorders and Schizophrenia (SADS), met criteria for severe to extreme anxiety or agitation, and Hendin et.al., [26] identified acute high affective intensity, in particular desperation, as the distinguishing feature of suicide completers in a case controlled psychological autopsy study.

In the course of our work on psychotic panic, [27] we have encountered a distinct psychopathologic state or syndrome related to panic and psychosis, [27,23] fitting with the findings of Hendin, Busch, and Snyder described above, which is reported by many suicide attempters as occurring immediately prior to their suicide attempt. In accordance with the aforementioned literature and our own observations, we have therefore hypothesized that this syndrome may serve as a "suicide trigger state" (ST state) mediating the transition to active suicide attempt in the potentially suicidal patient. Thus, identification of the proposed ST state in a highrisk population may be a powerful tool for the prediction of acute suicide risk.

Analysis of our data is suggestive of a state is marked by "ruminative flooding" (a confusing, uncontrollable and overwhelming profusion of negative thoughts) coupled with an acute, "frantic hopelessness", in which not only is there a fatalistic conviction that life cannot improve, but also an oppressive sense of entrapment and imminent doom. This builds to an intolerable, confused state in which patients feel that suicidal action is the only conceivable route of escape. In this state of severe distress, many patients have also reported the experience of "near-psychotic somatization" characterized by a concrete/somatic experience of thought, (e.g., thoughts creating head pressure) as well as somatic distortions (e.g., a subjective experience of a change in bodily size or shape).

In order to characterize the proposed ST state we have developed the Suicide Trigger Scale (STS), a rating scale that contains items testing for the above symptoms. Importantly, the STS does not rely on self-report of suicidal ideation. In this pilot study we aim to test the reliability and construct validity of the ST state as assessed by the STS-2, using statistical analysis of its coherence, internal structure, and relationship to a known validated instrument (the SCL-R 90). Further, we will assess the STS-2's relation to suicidal risk by examining the associations of scores on the scale and its individual components with a reported history of suicide attempt among patients with suicidal ideation.

\section{Methods \\ Participants}

The study was approved by the Beth Israel Institutional Review Board. Inclusion criteria were admission to psychiatric inpatient unit, chief complaint of suicidal wish/ ideation upon admission, age $\geq 18$ years, ability to understand and answer instrument questions, and literacy in the English language. The exclusion criteria were substance abuse in the 6 months prior to current hospitalization and a diagnosis of mental retardation or dementia. No other psychiatric diagnoses were exclusion criteria.

Subjects were recruited from the population of psychiatric patients receiving treatment at Beth Israel Medical Center's two non-dual diagnosis inpatient psychiatric units during the period of September 2006 through July 2008. During this time, of 2230 psychiatric admissions, a total of 141 (6.3\%) met inclusion criteria, agreed to participate, signed the informed consent forms and provided sufficient data to be used in the study. Of these 130 (92.2\%) completed all items on the STS-2 and 104 (73.8\%) also completed the SCL-90R. Suicide attempt history was considered definitive if it was confirmed by participants' clinicians' consensus recorded in the chart at the time of their discharge. Suicide attempt history is obtained by policy as part of the admission assessment for all psychiatric inpatients at Beth Israel Medical Center. Due to administrative issues unrelated to this project, only 41 charts were available for the retrospective review of suicidal ideation and behavior.

Demographic and clinical data are presented in Table 1. Axis I diagnosis was unavailable for 15 subjects due to unavailability of their charts for review. The demographic characteristics of our population are comparable to those of large clinical trials such as the STAR*D, $[28,29]$ demonstrating similar proportions of males and females and similar distributions of age and level of education, though in our sample a substantially higher percentage was identified as Hispanic while a lower percentage was identified as Caucasian. This difference reflects the demographics of the local population at large [30].

\section{Procedure and Instruments}

The participants were approached by research assistants who explained the purpose of the study, the nature of the scales, the measures taken to ensure confidentiality of the disclosed information and subjects' right to refuse or stop participation. After signing informed consent 
Table 1 Demographic and Clinical Variables

\begin{tabular}{|c|c|c|}
\hline & All subjects (total $\mathrm{N}=141$ ) & PCA subjects (total $\mathrm{N}=130$ ) \\
\hline \multicolumn{3}{|c|}{ Means and standard deviations of dimensional demographic variables } \\
\hline & Mean (SD) & Mean (SD) \\
\hline Age (range: 18-83) & $42.2(14.3)$ & $42.4(14.4)$ \\
\hline Years of education (range: 4-20) & $12.8(1.7)$ & $12.7(1.7)$ \\
\hline \multicolumn{3}{|c|}{ Frequencies and percentages of categorical demographic variables } \\
\hline & $N(\%)$ & $N(\%)$ \\
\hline \multicolumn{3}{|l|}{ Sex } \\
\hline Female & $85(60)$ & $77(59)$ \\
\hline Male & $56(40)$ & $53(41)$ \\
\hline \multicolumn{3}{|c|}{ Relationship status ( 2 subjects missing data) } \\
\hline Total w/o partner & $110(78)$ & 103(80) \\
\hline Single & $84(60)$ & $79(61)$ \\
\hline Divorced & $16(11)$ & $14(11)$ \\
\hline Widowed & $4(3)$ & $4(3)$ \\
\hline Separated & $6(4)$ & $6(5)$ \\
\hline Total w/Partner & $29(21)$ & $25(19)$ \\
\hline In committed relationship & $11(8)$ & $11(8)$ \\
\hline Married & $18(13)$ & $14(11)$ \\
\hline \multicolumn{3}{|l|}{ Race } \\
\hline Caucasian & $69(49)$ & $63(48)$ \\
\hline Hispanic & $48(34)$ & $44(34)$ \\
\hline Afro-American & $14(10)$ & $14(11)$ \\
\hline Other/missing & $6(4)$ & $5(4)$ \\
\hline Asian & $4(3)$ & $4(3)$ \\
\hline \multicolumn{3}{|c|}{ Axis I diagnosis (15 subjects missing data) } \\
\hline Total MDD & $43(30)$ & $41(31)$ \\
\hline MDD & $30(21)$ & $29(22)$ \\
\hline MDD with panic attacks & $13(9)$ & $12(9)$ \\
\hline Total bipolar & $31(21)$ & $27(20)$ \\
\hline Bipolar manic & $19(13)$ & $16(12)$ \\
\hline Bipolar depressed & $4(3)$ & $4(3)$ \\
\hline Bipolar mixed & $4(3)$ & $4(3)$ \\
\hline Bipolar with panic attacks & $3(2)$ & $3(2)$ \\
\hline Total psychotic & $29(21)$ & $27(20)$ \\
\hline Schizoaffective/Schizophrenia & $21(15)$ & $20(15)$ \\
\hline Psychosis NOS & $8(6)$ & $7(5)$ \\
\hline Total anxiety & $25(18)$ & $21(16)$ \\
\hline GAD with Panic Attacks & $24(17)$ & $20(15)$ \\
\hline Any diagnosis with panic attacks & $40(28)$ & $35(27)$ \\
\hline History of suicide attempt (SA) & $12(8.5)$ & $11(8.5)$ \\
\hline History of SA denied & $25(17.7)$ & $25(19.2)$ \\
\hline History of SA unknown & $105(74.5)$ & $95(73.1)$ \\
\hline
\end{tabular}

forms, subjects were given the self-administered STS-2 and SCL-90R to complete. The scales were administered in no particular order. Research volunteers collected demographic information from patient charts after the questionnaires were completed. Diagnoses and medication information were obtained from the medical charts of the psychiatric unit.
Suicide Trigger Scale version 2 (STS-2)

The STS-2 (additional file 1 ) is a 39 item scale with 3 response categories $(0=$ not at all, $1=$ somewhat, $2=\mathrm{a}$ lot) and is derived from STS-1 [31]. The STS-1 was originally given to 36 subjects on the same acute psychiatric units as STS-2 and re-administered 7-14 days later to those 13 who were still hospitalized (Cronbach's 
alpha 0.86;test re-test reliability 0.911)[31]. The scores had normal distribution. Exploratory factor analysis with the STS-1 revealed 4 factors with eigenvalues greater than 1. These were labeled Dread and Doom (Factor 1), Changes in Body (Factor 2), Head Pressure (Factor 3), and Hopelessness (Factor 4). After a consensus development meeting, the STS-1 was then revised by removing non-contributory items and adding new clinicallyderived items to capture more symptoms of dissociation, somatization, head pain, and dread. The result was the 39-item STS-2.

\section{The Symptom Checklist -90-Revised (SCL-90-R)}

The SCL-90-R is a well-established 90-item scale with 5 response categories $(0=$ 'not at all' to $4=$ 'very much') that assesses the presence and intensity of a wide variety of psychological symptoms [32]. The total score and 9 sub-scales were used in the analyses. The sub-scales of the SCL-90-R are Anxiety, Depression, ObsessiveCompulsive, Interpersonal Sensitivity, Somatization, Phobic Anxiety, Psychoticism, Hostility, and Paranoid Ideation, and have all been found to have high reliability with Cronbach's alphas ranging from 0.8 to 0.9 , oneweek test-retest reliability ranging from 0.8 to 0.9 , and convergent validity with the Minnesota Multiphasic Personality Inventory (MMPI) [32]. Item 59, which assesses the presence of "thoughts of death," was also used in the analysis.

\section{Statistical Analysis}

Reliability was assessed through Cronbach's alpha, which was used as a measure of internal consistency. Construct validity was assessed through a variety of statistical methods, including principal component analysis to explore the internal structure of the STS, Receiver Operator Characteristic (ROC) analysis with Fisher's exact test for cut-score to demonstrate clinical significance, and logistic regression analysis to examine which items of the STS-2 appeared to be most associated with suicidal action. Additionally, concurrent validity was assessed with correlation coefficients between STS-2 and SCL-90R scores and sub-scores.

\section{Internal Structure of the STS-2}

Principal components analysis (PCA) with component rotation was used to assess the internal structure of the STS[33]. Because PCA requires pairwise-complete observations to calculate the correlation matrix that determines the factor loadings only data from those subjects $(\mathrm{N}=130)$ who completed every item of the STS-2 could be used. (See Table 1 for comparison of PCA subjects and the total sample.) Three methods were used in succession to decide the number of components to be extracted in PCA: on first pass, eigenvalues $>1$, on second pass Scree plot, and finally, interpretability of components was used to eliminate components marginal on scree plot.

Following PCA, component rotation was performed by both Varimax rotation and Promax rotation, both with Kaiser Normalization. Varimax rotation preserves orthogonality of components while maximizing the variance of factor loadings on each component. The aim of this technique is to produce conceptually coherent, maximally independent, component subscales. Promax rotation does not preserve orthogonality, but aims to maximize component coherence and thus their semantic interpretability.

\section{Clinical Significance of the STS-2 - Construct Validity}

Clinical significance of the STS-2 was assessed using ROC analysis of the STS-2 scores in discriminating past suicide attempters from those who had not made any suicide attempts[34]. ROC was performed on the unscaled STS to determine both Area Under the Curve (AUC) as a measure of the scale's robustness, and an optimal cut-score, the statistical significance of which was measured using Fisher's exact test. As the distributions of STS-2 scores in the PCA group and the subgroup chart-reviewed for suicide attempt history were very close (mean(standard deviation); 38(18) vs. 42(15), respectively), ROC analysis was also performed on the principal components produced in the Varimax PCA analysis to measure their robustness as discriminators between suicide attempters and non-attempters.

In addition, logistic regression analysis[35] was used to assess which individual items appeared to be most strongly associated with suicidality. Logistic regression analysis was used to produce a coefficient for each item of the STS-2 based on a separate regression of SA onto it. The resulting odds ratio is interpreted as the change in log-odds of SA when that item score increases by one.

\section{Concurrent Validity}

Finally, scores on the STS-2 and its principal components were correlated with total and subscale scores on the SCL-90R as a measure of concurrent validity. Bonferroni correction for multiple $(\mathrm{n}=30)$ comparisons was used to correct the threshold for statistical significance.

\section{Results}

The scale showed a normal distribution of scores (pvalues of the Shapiro-Wilk test of normality were 0.974 and 0.18 for the SA and non-SA groups respectively). For the 130 subjects who completed the STS-2, there was a mean score of 34 and standard deviation of 16 .

\section{Reliability}

The STS-2 showed high internal consistency with a Cronbach's alpha of 0.949 . Four items (\#13 trouble falling asleep, 
\#16 panic attack, \#29 ideas turning over and over, and \#30 feeling doomed) were demonstrated to decrease Cronbach's alpha. Of these only one, 'doom', loaded strongly on our final principal component solution (see Table 2).

\section{Internal Structure}

Principal component analysis extracted 8 components with eigenvalues $>1$, together accounting for $66 \%$ of the variance in the STS scores. The Scree plot suggests the use of one to three principal components (see Figure 1). However, the one-component solution lacked semantic coherence, while the three-component solution yielded two components approximately equivalent to the two-component solution followed by a minimally contributory and semantically incoherent third component. Thus the solution with two principal components accounting for $44 \%$ of the variance $(37 \%$ and $7 \%$, respectively), was found to best fit the data and was used as the basis for subsequent analysis.

Based on the two factor solution, we characterized the two principal components as follows:

Principal Component 1: Ruminative Flooding (thought experienced as a confusing and uncontrollable of flood of ruminative ideas) and Near-Psychotic Somatization (distorted/bizarre somatic perception and concrete/somatic experience of thought).

Principal Component 2: Frantic Hopelessness (acute, fatalistic conviction that one's situation is hopeless and life cannot improve compounded by a fearful and oppressive sense of entrapment and doom).

The Varimax solution, which maintains component orthogonality, is very similar to the Promax solution presented here in Table 2. Inspection of the graphs of

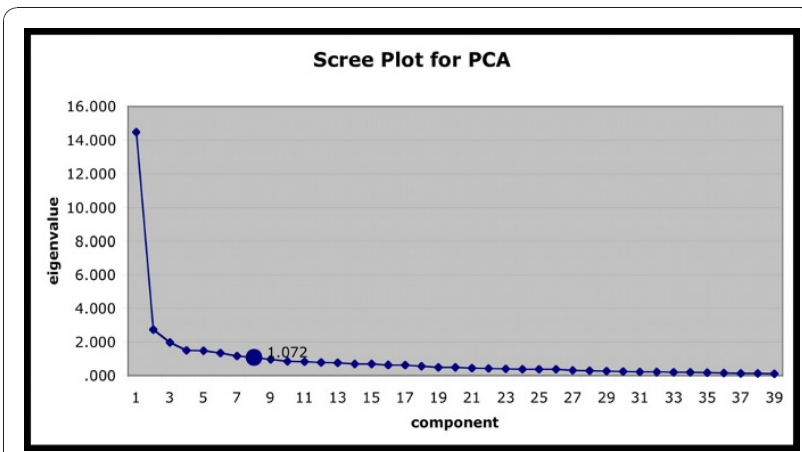

Figure 1 Scree Plot for PCA. The eigenvalue for each component generated by first-pass principal component analysis. Eight components had an eigenvalues $>1$.

ordered factor loadings suggested an item loading cut-off value of 0.6 for both principal components (see Figure 2). The graphs show clusters of items loading similarly on a given factor, and inspection of items with similar loading values reveals generally similar content. Items describing a sense of entrapment (\# 4,14,26,36) had substantial loadings (0.4-0.6) on both components but did not meet the cut-off threshold.

\section{Clinical significance - Construct Validity}

ROC analysis of the STS-2 raw scores $(\mathrm{N}=36)$ showed significant and robust detection of a reported history of suicide among suicidal ideators with an AUC of 0.724 and asymptotic significance of 0.027 . Analysis of the ROC curve suggests an optimal cut-score of 48 (approximately one standard deviation above the sample mean). Sensitivity for a cut-off total STS-2 score of 48 is 0.667 , specificity is 0.704 and the 1 -sided p-value of the

Table 2 Two-component solution: Promax rotation with Kaiser normalization

\begin{tabular}{|c|c|c|}
\hline STS-2 numbered items & Component 1 factor loadings & Component 2 factor loadings \\
\hline 18. Strange sensations in body or on skin & .872 & \\
\hline 19. Something happening to body & .847 & \\
\hline 39. Headache from too many thoughts in head & .808 & \\
\hline 5. Unusual physical sensations & .797 & \\
\hline 20. Thoughts racing & .779 & \\
\hline 21. Have no control & .743 & \\
\hline 37. Pressure in head from thinking too much & .731 & \\
\hline 6. Head could explode from too many thoughts & 699 & \\
\hline 11. Head or body parts changed in size or shape & .658 & \\
\hline 30. Doomed & & .741 \\
\hline 1. Wake up tired and not refreshed & & .739 \\
\hline 32. Would like troubling thoughts to go away but they won't & & .737 \\
\hline 34. Hope of change (reversed) & & 679 \\
\hline 23. Think things will be normal again (reversed) & & .676 \\
\hline
\end{tabular}



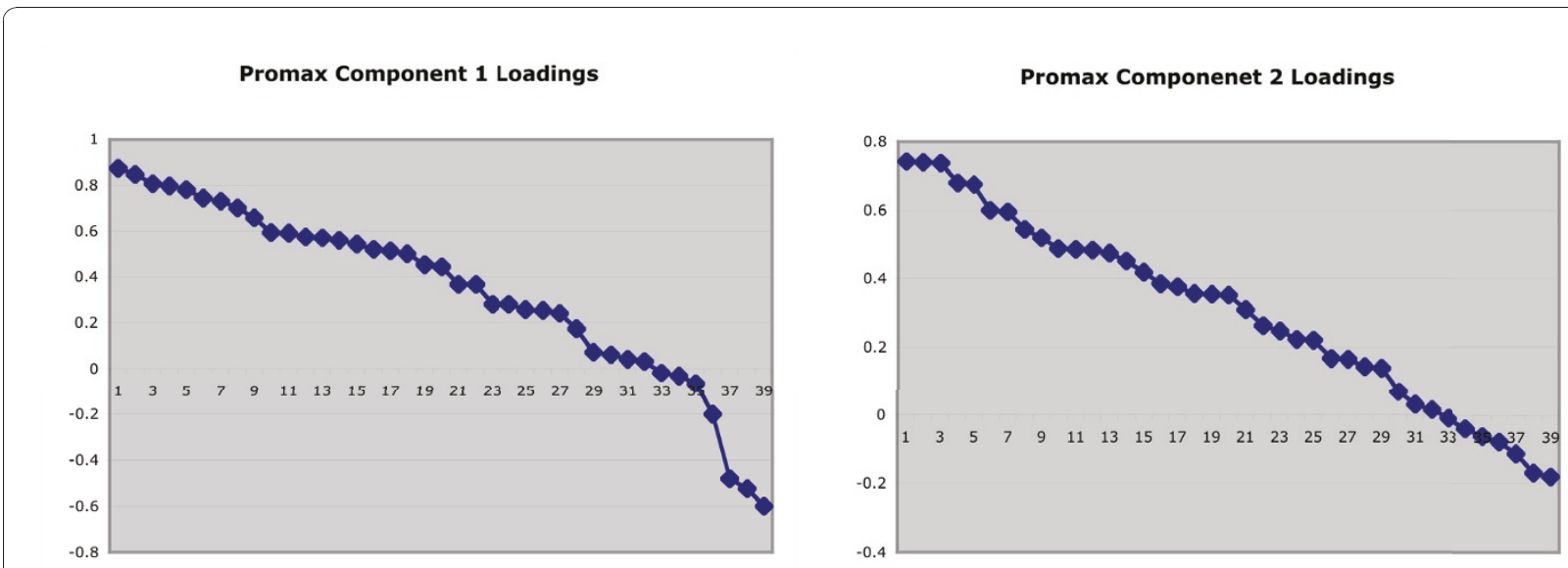

Figure 2 Ordered factor loadings for the STS-2 individual items on principal components.

Fisher exact test is significant at the 0.02 level (see Figure 3).

\section{ROC analysis of subscales}

ROC analysis of both Promax and Varimax 2-component solutions found statistically significant (asymptotic $\mathrm{p}=0.002)$ prediction of suicide attempt history in the second component, (Frantic Hopelessness) with AUCs of 0.83 and 0.82 , respectively. This finding correlates well with the results of the logistic regression on the individual items discussed below.

\section{Regression analysis}

Logistic regression was performed to determine the association between each STS-2 item and the reported

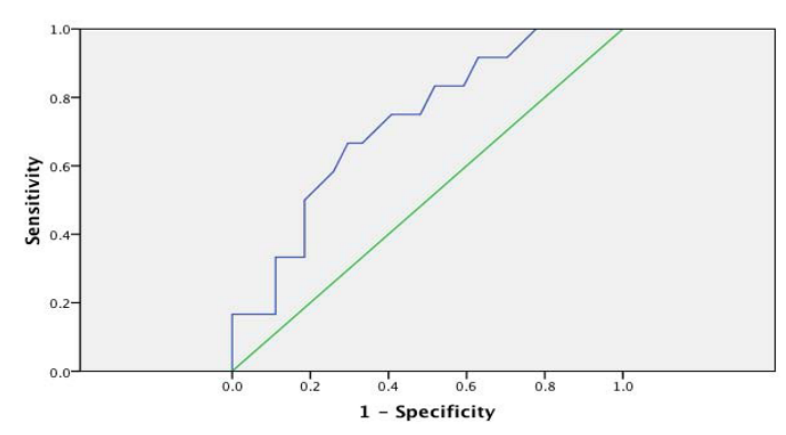

Figure 3 The ROC curve for the global score on the STS-2. The ROC Curve (blue) and reference line (green) for the STS-2 shows the sensitivity (probability of a true positive being detected) versus 1 specificity (probability of false positive) for the scale in identifying subjects with history of SA using incrementally decreased cut-off scores. Diagonal segments are produced by ties. The point of greatest separation between the ROC curve and the reference line marks the sensitivity (.667) and specificity (.774) of the optimal cut-off score. history of suicide attempt $(\mathrm{N}=36)$. Regression coefficients and uncorrected p-values for STS-2 individual items regressed onto reported history of SA are presented in Table 3. Although logistic regression analysis of the individual items of the STS-2 against history of SA found no statistically significant results after Bonferroni correction for multiple comparisons (required p value $<0.00128$ ), this criterion may be excessively stringent [36]. The items with the highest coefficients were all descriptive of one of three themes: ruminative flooding, doom/hopelessness, and entrapment. Item \#33 (can stop thoughts that are troubling) had the highest odds ratio (16.01). In other words, subjects who endorsed a score of 2 ("a lot") were approximately 16 times more likely to have had a previous suicide attempt than subjects who endorsed a score of 1 ("somewhat"). Likewise, 9 items describing ruminative flooding (Items \#2, 3, 9, 12, 13, 20, 29, 32, and 33) had a mean regression coefficient of 0.97 (corresponding to an OR of 2.64). Contrary to expectations, items describing near-psychotic somatization (Items \#5, 11, 18,19 and 24) produced negative coefficients in the regression analysis (albeit only at an uncorrected trend level of significance). Thus in our sample population of psychiatric inpatients, more bizarre somatic experience corresponded to a decreased likelihood of having made a past suicide attempt.

\section{Integration of Principal Component and Regression Analyses}

Several of the best-performing items in regression analysis loaded strongly (factor loading values $\geq 0.5$ ) on the principal components. Furthermore, items with relatively high regression coefficients $(>1.0)$ had a strong mean loading of 0.46 on Principal Component 2 (which was a robust detector of 
Table 3 Regression coefficients and uncorrected p-values for STS-2 individual items regressed onto reported history of SA

\begin{tabular}{|c|c|c|}
\hline STS-2 numbered items & Regression coefficient & p-value \\
\hline 33. Can stop thoughts that are troubling (reverse scored) & 2.77 & 0.01 \\
\hline 4. No exit & 2.42 & 0.03 \\
\hline 30. Doomed & 2.02 & 0.01 \\
\hline 36. No escape & 1.96 & 0.02 \\
\hline 28. Sense of dread & 1.76 & 0.02 \\
\hline 38. Think you will ever feel better (reverse) & 1.69 & 0.03 \\
\hline 9. Hard to stop worrying & 1.57 & 0.01 \\
\hline 13. Trouble falling asleep because of thoughts you cannot control & 1.54 & 0.02 \\
\hline 17. Expect the worst & 1.49 & 0.07 \\
\hline 34. Hope of change (reverse) & 1.45 & 0.01 \\
\hline 23. Think things will be normal again (reverse) & 1.42 & 0.01 \\
\hline 26. Trapped & 1.39 & 0.02 \\
\hline 35. Something horrible going to happen & 1.20 & 0.05 \\
\hline 12. Cannot concentrate or make decisions due to too many thoughts & 1.05 & 0.05 \\
\hline 32. Would like troubling thoughts to go away but they won't & 1.05 & 0.07 \\
\hline 16. Sudden panic-attack or physical symptoms & 0.94 & 0.12 \\
\hline 15. World feels different & 0.71 & 0.14 \\
\hline 1. Wake up tired and not refreshed & 0.68 & 0.17 \\
\hline 27. Feel blood rushing through veins & 0.66 & 0.17 \\
\hline 25. Helpless to change & 0.64 & 0.23 \\
\hline 14. World closing in & 0.59 & 0.26 \\
\hline 7. Ordinary things look strange or distorted & 0.56 & 0.33 \\
\hline 29. Ideas turning over and over, won't go away & 0.55 & 0.35 \\
\hline 10. Hopeless & 0.41 & 0.43 \\
\hline 20. Thoughts racing & 0.33 & 0.50 \\
\hline 6. Head could explode from too many thoughts & 0.29 & 0.56 \\
\hline 21. Have no control & 0.19 & 0.69 \\
\hline 8. Worry bad things might happen & 0.19 & 0.72 \\
\hline 2. Thoughts confused & -0.02 & 0.98 \\
\hline 3. Many thoughts in head & -0.10 & 0.89 \\
\hline 22. Bothered by thoughts that do not make sense & -0.11 & 0.82 \\
\hline 31. Something wrong physically & -0.11 & 0.81 \\
\hline 39. Headache from too many thoughts in head & -0.24 & 0.59 \\
\hline 5. Unusual physical sensations & -0.46 & 0.32 \\
\hline 19. Something happening to body & -0.62 & 0.15 \\
\hline 37. Pressure in head from thinking too much & -0.87 & 0.11 \\
\hline 18. Strange sensations in body or on skin & -0.92 & 0.07 \\
\hline 24. Sensations you cannot describe & -1.14 & 0.06 \\
\hline 11. Head or body parts changed in size or shape & -1.41 & 0.06 \\
\hline
\end{tabular}

past SA), but a weak mean loading (0.15) on Principal Component 1 (which performed poorly as a detector of past SA under ROC analysis). In combination with the heavy loading of somatic symptoms on Component 1 , this appears to account for Component 1's poor performance as a predictor of suicide attempt history on ROC analysis.
Concurrent and External validity of the STS-2

One hundred and four (104) subjects completed both the SCL-90-R and the STS-2. Correlations between STS-2 total score and principal component 1 and 2 scores were calculated and correlated with the SCL-90-R total scores, the nine subscales and Item 59 - 
"Thoughts of death or dying". There was a high correlation between total scores on the STS-2 and the SCL-90; $\mathrm{r}=0.77$. High correlations were found for all subscales, principally for depression and anxiety. The lowest correlation coefficient was found for Item 59. However this is most likely an artifact of the low range of scores possible for a single item as compared to a subscale, which makes it more susceptible to noise. The results are shown in Table 4 below. All correlations were significant to $\mathrm{p}<0.001$, (equivalent to $\mathrm{p}<0.03$ after Bonferroni correction for multiple comparisons).

Substantial numbers of high STS-2 scores were found in all demographic and diagnostic subgroups, demonstrating that the instrument measures a state that is not demographically bound, and is distinct from panic, mood, and psychotic disorder. Table 5 shows the mean scores on the STS-2 across demographic and diagnostic variables as well as the percentage and $\mathrm{N}$ of each demographic subgroup of the entire sample that scored above the cut-score. While substantial differences may be noted between different demographic subgroups, a substantial proportion $(>20 \%)$ of each subgroup reported a score greater than the cutscore. Comparison of demographic and diagnostic categories by Fisher exact test demonstrated no significant differences at the $\mathrm{p}<0.05$ level, providing preliminary evidence of external and divergent validity.

\section{Discussion}

The results of this preliminary investigation are limited by its retrospective design, reliance on self-report, relatively small size of the whole sample and of an even smaller subgroup of subjects with data on past suicide attempts. Thus, our findings should be viewed as exploratory in nature and are not intended to demonstrate causality or define a definitive component structure. Nonetheless, the high Cronbach's alpha suggests that the STS-2 defines a coherent psychopathological clinical state, and principal component analysis, though
Table 5 STS-2 Scores by demographic subgroup

\begin{tabular}{lcc}
\hline Demographic & $\begin{array}{c}\text { STS score: Mean } \\
\text { (SD) }\end{array}$ & $\begin{array}{c}\mathbf{N}(\%) \text { with score > } \\
\mathbf{4 8}\end{array}$ \\
\hline $\begin{array}{l}\text { Sex } \\
\text { Female }\end{array}$ & $39.8(17.6)$ & $32(38)$ \\
Male & $36.4(17.6)$ & $16(29)$ \\
\hline Race & & \\
\hline Caucasian & $36.1(14.75)$ & $21(30)$ \\
Hispanic & $34.8(18.6)$ & $20(42)$ \\
African-American & $29.1(13.8)$ & $3(21)$ \\
\hline Primary Axis I diagnosis & & \\
\hline \multicolumn{1}{l}{ MDD } & $36.1(15.0)$ & $12(28)$ \\
$\quad$ Bipolar & $32.1(17.6)$ & $9(29)$ \\
$\quad$ Psychotic & $32.6(15.2)$ & $9(31)$ \\
$\quad$ Anxiety D/O with panic & $35.5(17.2)$ & $11(45)$ \\
$\quad$ attacks & & $17(44)$ \\
\hline Total With Panic Dx in Axis I & $38.6(16.1)$ & $23(23)$ \\
Total Without Panic Dx in & $32.2(15.8)$ & $8(67)$ \\
Axis I & & $8(32)$ \\
\hline History of SA & & \\
No History of SA & $44.45(11.1)$ & \\
\hline & $36.4(14.2)$ & \\
\hline
\end{tabular}

underpowered by a factor of two, is suggestive of two principal components.

The first component was termed Ruminative Flooding and Near-Psychotic Somatization, while the second was termed Frantic Hopelessness. Items describing entrapment and dread loaded strongly though below the cut-off level for both components, and were found in regression analysis to be highly sensitive to past SA. We conceptualize entrapment and dread as elements of Frantic Hopelessness. High scores on the STS-2 demonstrated significant sensitivity and specificity in distinguishing suicidal ideators with a history of attempt from those without. Finally there were high correlations between scores on the STS-2 and the SCL-90-R assessment of general psychopathology, as well as the depression and anxiety subscales of the SCL90$\mathrm{R}$, consistent with the conception of the suicide trigger

Table 4 Correlation coefficients ( $r$ ) between STS-2 scores and SCL-90 sub-scale scores

\begin{tabular}{llll}
\hline & $\begin{array}{l}\text { STS-2 } \\
\text { total score }\end{array}$ & Principal comp. 1 score & Principal comp. 2 score \\
\hline SCL5- Anxiety & 0.79 & 0.80 & 0.79 \\
\hline SCL4- Depression & 0.71 & 0.75 & 0.72 \\
\hline SCL2- Obsessive Compulsive & 0.69 & 0.70 & 0.71 \\
\hline SCL3-Interpersonal Sensitivity & 0.67 & 0.67 & 0.68 \\
\hline SCL1- Somatization & 0.63 & 0.64 & 0.65 \\
\hline SCL7- Phobic Anxiety & 0.62 & 0.62 & 0.63 \\
\hline SCL9 -Psychoticism & 0.61 & 0.61 & 0.63 \\
\hline SCL6- Hostility & 0.56 & 0.55 & 0.57 \\
\hline SCL8- Paranoid Ideation & 0.53 & 0.51 & 0.55 \\
\hline Item 59- Thoughts of death & 0.53 & 0.58 & 0.55
\end{tabular}


state as a syndrome of disordered thought and affect. Our findings appear to be the first quantitative description of a discrete psychopathologic state other than suicidal ideation, and distinct from Axis I diagnosis, that demonstrates a differential association with suicidal action.

Our data supports our hypothesis that this state is associated with suicidal action, but cannot demonstrate causality. Further investigation is warranted to determine whether this state indeed serves as an acute trigger state for suicidal actions or, alternatively, serves as a marker of a trait susceptibility to taking suicidal action. Our results indicate that items encoding Ruminative Flooding and Frantic Hopelessness, including those describing entrapment and dread, were particularly associated with history of suicide attempt and thus may play a more prominent mediating role in the precipitation of suicidal action.

Combining the results from all our statistical analyses, our data paint a picture of a panic-like state characterized by disturbed thought process (rumination, perceptual distortion, near-psychotic somatization), and a pathological cathexis of thought content and affective arousal which we term 'frantic hopelessness.' In this state, hopelessness is acutely sharpened to a sense of doom, entrapment and dread.

The robustness of the second principal component of the STS-2 (Frantic Hopelessness) in distinguishing ideators with history of attempt from those without is consistent with the literature that identifies hopelessness as a primary risk factor for suicide attempt[37]-[38-40]. It might be argued that indeed our results no more than recapitulate Beck's finding that hopelessness is a strong predictor of suicidality. We suggest however that the coherence of the STS-2 demonstrated by its high Cronbach's alpha combined with the scale's inclusion of many items which are clearly distinct from hopelessness on face value, argues for a unique clinical syndrome broader in scope than hopelessness alone as described by Beck. Furthermore, the second principal component, while including elements akin to canonically described hopelessness, is distinct not only by virtue of existing within the context of this syndrome, but also because it contains items - such as doom (\#30), fatigue (\#1), and cognitive oppression (\#32) - which lend it an acute, fatalistic and oppressive quality not previously described. This finding however is limited by lack of power for a definitive factor analysis.

Though Cronbach's alpha was high, two items, doom (\#30) and panic attacks (\#16) reduced this metric. That Cronbach's alpha was decreased by item 30 "Doom" could suggest that doom does not belong to the syndrome. However, Cronbach's alpha was not decreased by semantically similar items, or by other items that loaded most heavily on the Frantic Hopelessness component. An alternative explanation may be that 'doom', a somewhat literary word, was not familiar in the vocabulary of some subjects, and perhaps more so given the high proportion of Hispanic subjects, many of whom may not have been raised in an English-speaking environment. Similarly, item 16 "panic attack" may have reduced Cronbach's alpha because it relies upon subject familiarity or comfort with this technical term, which may not be as common in the lay vocabulary as, for example, "depression." Further, the high correlation of the total STS-2 scores and the two principal components with the SCL90-R Anxiety Subscale is consistent with the literature supporting panic and anxiety disorders as risk factors for suicide attempt $[23,41,42,4]$.

Our finding that those items in the first principal component which are descriptive of Ruminative Flooding (such as racing and too many thoughts) generally produced fairly high regression coefficients (mean value 0.97) is consistent with the findings of Morrison and O'Connor[19,43] who identify ruminative thought as a suicide risk factor. The high correlation between STS-2 and SCL-90R total scores is in agreement with the literature that finds general severity of psychopathology to be a risk factor for suicide[4,44,45].

The marked variability of SCL-90R Item 59 (thoughts of death or dying) in a sample population of patients presenting with SI highlights the limited reliability of patient self report of SI. The comparatively low correlation between scores on item 59, which should, a priori, be high for suicidal ideators, and scores on the STS-2 items most predictive of past SA as grouped in Component 2 , highlights the importance of a clinical measure which does not rely on overt self-report of suicidality.

Our results also present the unexpected finding that items of the STS-2 that describe near-psychotic somatization (which could be interpreted as variants of somatic and dissociative symptoms of panic attack) appear to correlate negatively - though not significantly - with history of SA. This is contrary to the literature linking suicide risk to panic attacks, and overall severity of psychopathology and psychoticism $[21,24,45]$. While our data are not sufficiently powered to demonstrate this, inspection of score distributions across different axis I diagnoses suggests that schizoaffective subjects were more heavily represented among those with history of SA but had lower scores on the STS-2 somatization items, while subjects scoring highest on somatization items were rather those with combined depression and anxiety diagnoses. Possibly this is merely an artifact of small sample size and sample population. We speculate however, that among those subjects with primary anxiety diagnoses, somatization is a marker of concern for bodily integrity (as in the hypochondriac) and may protect against self-harm behaviors $[46,47]$. 
As highlighted, our study has a number of limitations. In summary, while the study has the advantage of comprising a demographically and diagnostically balanced population, it is limited in sample size and was not sufficiently powered to reliably detect differences between subgroups. Furthermore, the sample size is too small for a definitive factor analytic study and thus the factor structure should be considered preliminary. The limitations imposed on the secondary analyses by small sample size were magnified by the lack of availability of complete clinical data for many subjects due to lack of chart availability, such that Axis I diagnosis unknown for 15 subjects and suicide attempt history was only known for 39 subjects. Though there were no significant differences between the subject group as a whole and the subgroup of subjects whose charts were available for review of SA history in terms of ethnic group composition, or scores on the STS-2, a significantly higher proportion of the entire group carried bipolar and psychotic disorder diagnoses than in the chart-reviewed subgroup (approximately $40 \%$ vs. $25 \%, \mathrm{p}=0.04$ ). The cultural diversity of the sample may also affect the results in ways which the current study is unable to account for due to cultural mediation of symptomatology; somatic symptoms in particular may exhibit culturally mediated differences in salience, semantic significance, and prognostic value $[48,49]$. A further limitation common to studies of infrequent phenomena such as suicide is its retrospective design, and, in particular, its reliance on self-report as the only measure of suicide attempt history. As with all self-report instruments, there is risk that subjects did not understand all of the scale items, answer accurately, or without bias.

\section{Conclusions}

Within the study limitations, our findings suggest that the STS-2 describes a novel and coherent syndrome of psychic experience, separate from suicidal ideation and DSM-IV axis I diagnosis, which demonstrates an association with report of past suicidal action. This state consists of ruminative flooding, near-psychotic somatization and frantic hopelessness. Scores on the STS-2 can distinguish between suicidal ideators who report having made an attempt in the past from those who deny past suicide attempts.

There is a great need for a reliable and valid instrument that would enable health care professionals to identify patients at increased risk of acting on their ideations and to pre-empt serious suicide attempts, particularly in those patients at greater risk for "low plan" or impulsive suicide or those who deliberately conceal or unconsciously repress suicidal ideation[14,15]. Thus, an assessment that does not rely heavily on the selfreported cognitions of patients would be of particular value. The lack of emphasis on suicidal ideation and plan in the STS-2 could make it particularly suited to this task, as these features may be absent, outside of conscious awareness, or may be intentionally underreported. Future larger studies utilizing prospective approaches, larger samples, and corroborated suicidal events are therefore needed to substantiate the current results and establish the STS-2 as a predictor of suicidal action. Future studies should also explore the influence of culture, gender, and primary psychiatric diagnosis on STS global scores and subscales, to demonstrate its ability to predict suicide acutely and prospectively and to further elucidate which elements of the state are most predictive of suicide attempts.

\section{Additional material}

Additional file 1: STS-2 PDF.

\section{Acknowledgements}

We would like to acknowledge the substantial efforts of the research volunteers who collected and tabulated the data for this study, Serena Fox MD who helped coordinate their efforts, and Ramin Mojtabai MD, PhD, MPH for his invaluable counsel and editorial support in the drafting of the manuscript.

This research was supported in part by the Hope for Depression Research Foundation, the Empire Clinical Research Investigator Program, the Family Center for Bipolar Disorder, and the Zirinsky Mood Disorders Center. This research was presented in part at the following meetings: Yaseen Z, Johnson M, Galynker I. Construct Validity of a Suicide Trigger State. The 162nd Annual Meeting of the American Psychiatric Association, San Francisco, CA (2009)

Yard S, Tecuta L, Blumenfeld A, Mojtabai R, Cohen L, Galynker I: Reliability and Validity of the Para-Psychotic Symptoms Scale. The 160th Annual Meeting of the American Psychiatric Association, San Diego, CA (2007)

\section{Author details}

${ }^{1}$ Beth Israel Medical Center, New York, New York, USA. ${ }^{2}$ Teachers College, Columbia University, New York, New York, USA. ${ }^{3}$ National Institute of Mental Health, Bethesda, Maryland, USA.

\section{Authors' contributions}

ZY drafted the manuscript and contributed the design and completion of the data analyses. CK assisted in the drafting of the manuscript, performance of the statistical analyses, as well as the coordination of the study. MSJ designed and performed the principal statistical analyses. DE and LJC provided substantial editorial input in the drafting of the manuscript. IIG conceived of the study, and participated in its design and coordination and helped to draft the manuscript. All authors read and approved the final manuscript.

\section{Competing interests}

The authors declare that they have no competing interests.

Received: 12 June 2010 Accepted: 14 December 2010

Published: 14 December 2010

\section{References}

1. Brown GK, Ten HT, Henriques GR, Xie SX, Hollander JE, Beck AT: Cognitive therapy for the prevention of suicide attempts: a randomized controlled trial. JAMA 2005, 294:563-70.

2. Mann JJ, Waternaux C, Haas GL, Malone KM: Toward a clinical model of suicidal behavior in psychiatric patients. Am J Psychiatry 1999, 156:181-189. 
3. Motto JA, Bostrom A: Empirical indicators of near-term suicide risk. Crisis 1990, 11(1):52-9.

4. Hall RCW, Platt DE, Hall RCW: Suicide risk assessment: A review of risk for suicide in 100 patients who made severe suicide attempts. Evaluation of suicide risk in a time of managed care. Psychosomatics 1999, 40:18-27.

5. Pfaff JJ, Acres JG, McKelvey RS: Training general practitioners to recognize and respond to psychological distress and suicidal ideation in young people. Med J Aust 2001, 174(5):222-6.

6. Pfaff JJ, Almeida OP: Detecting suicidal ideation in older patients: identifying risk factors within the general practice setting. $\mathrm{Br} J$ Gen Pract 2005, 55(513):269-73.

7. Oquendo MA, Currier D, Mann JJ: Prospective studies of suicidal behavior in major depressive and bipolar disorders: what is the evidence for predictive risk factors? Acta Psychiatr Scan 2006, 114(3):151-8.

8. Niméus A, Hjalmarsson Ståhlfors F, Sunnqvist C, Stanley B, TräskmanBendz L: Evaluation of a modified interview version and of a self-rating version of the Suicide Assessment Scale. Eur Psychiatry 2006, 21(7):471-7, Epub 2006 Mar 20.

9. Niméus A, Alsén M, Träskman-Bendz L: The suicide assessment scale: an instrument assessing suicide risk of suicide attempters. Eur Psychiatry 2000, 15(7):416-23.

10. Holmstrand C, Nimeus A, Traskman-Bendz L: Risk factors of future suicide in suicide attempters - a comparison between suicides and matched survivors. Nord J Psychiatry 2006, 60(2):162-167.

11. Beck AT, Kovacs M, Weissman A: Assessment of suicidal intention: the Scale for Suicide Ideation. Journal of Consulting and Clinical Psychology 1979, 47:343-352.

12. Harriss $L$, Hawton $K$ : Suicidal intent in deliberate self-harm and the risk of suicide: the predictive power of the Suicide Intent Scale. J Affect Disord 2005, 86(2):225-233.

13. Motto JA, Bostrom A: Empirical indicators of near-term suicide risk. Crisis $1990,1: 52-9$

14. Horesh N, Zalsman G, Apter A: Suicidal behavior and self-disclosure in adolescent psychiatric inpatients. J Nerv Ment Dis 2004, 192(12):837-42.

15. Busch KA, Fawcett J, Jacobs DG: Clinical correlates of inpatient suicide. $J$ Clin Psychiatry 2003, 64(1):14-9.

16. Deisenhammer EA, Ing CM, Strauss R, Kemmler G, Hinterhuber H, Weiss EM: The duration of the suicidal process: how much time is left for intervention between consideration and accomplishment of a suicide attempt? J Clin Psychiatry 2009, 70(1):19-24, Epub 2008 Oct 21.

17. Esposito C, Sirito A, Boergers J, Donaldson D: Affective, behavioral, and cognitive functioning in adolescents with multiple suicide attempts. Suicide Life Threat Behav 2003, 33:389-399.

18. Nock MK, Kazdin AE: Examination of affective, cognitive, and behavioral factors and suicide-related outcomes in children and young adolescents. Journal of clinical child and adolescent psychology 2002, 31:48-58.

19. Morrison R, O'Connor RC: A systematic review of the relationship between rumination and suicidality. Suicide Life Threat Behav 2008, 38(5):523-38

20. Rudd MD, Joiner T, Rajab MH: Treating suicidal behavior: an effective time-limited approach. New York; Guilford Press; 2001

21. Randomsky ED, Haas GL, Mann JJ, Sweeny JA: Suicidal behavior in patients with schizophrenia and other psychotic disorders. American journal of psychiatry 1999, 156:1590-1595.

22. Goodwin RD, Hamilton SP: Panic as a marker of core pathological processes. Pyschopathology 2001, 34:278-288.

23. Katz CE, Mojtabai R, Camacho K, Samuel J, Galynker I: Panic attack and suicide risk: A multivariate logistic regression analysis of the National Epidemiological Survey of Alcoholism and Related Conditions. Poster session presented at the 162 nd annual meeting of the American Psychiatric Association, San Francisco, CA; 2009.

24. Weissman MM, Klerman GL, Markowitz JS, Ouellette R: Suicidal ideation and suicide attempts in panic disorder and attack. N Engl J Med 1989, 231:1209-1214

25. Schnyder U, Valach L, Bichsel K, Michel K: Attempted suicide: Do we understand patients reasons? General hospital psychiatry 1999, 21:62-69

26. Hendin $\mathrm{H}$, Maltsberger JT, Haas AP, Szanto K, Rabinowicz H: Desperation and other affective states in suicidal patients. Suicide Life Threat Behav 2004, 34(4):386-94.

27. Galynker I, leronimo C, Perez-Aquino A, Lee Y, Winston A: Panic attacks with psychotic features. Journal of Clinical Psychiatry 1996, 57:402-406.
28. Fava M, Rush AJ, Trivedi MH, et al: Background and rationale for the Sequenced Treatment Alternatives to Relieve Depression (STAR*D) study. Psychiatr Clin North Am 2003, 26:457-494.

29. Rush AJ, Fava M, Wisniewski SR, et al: Sequenced treatment alternatives to relieve depression (STAR*D): rationale and design. Control Clin Trials 2004, 25:119-142.

30. NYC Census. 2007 [http://www.nyc.gov/html/dcp/html/census/popacs. shtml], Accessed April 15, 2009.

31. Galynker I, Mojtabai R, Cohen L: Panic attacks with psychotic features: are they "non-affective acute remitting psychosis?". Symposium 575 presentaed at the 159th Annual Meeting of the American Psychiatric Association, Toronto, Canada; 2006.

32. Goldman RS, Robinson D, Grube BS, Hanks RA, Putnam K, Walder DJ, Kane JM: General Psychiatric Symptoms Measures. In Handbook of Psychiatric Measures. 1 edition. Edited by: The Taskforce for the Handbook of Psychiatric Measures. Washington DC: American Psychiatric Association Press; 2000:81-84.

33. Hyvarinen A, Karhunen J, Oja E: Independent component analysis New York: Wiley; 2001.

34. Fawcett T: An introduction to ROC analysis. Pattern Recognition Letters 2006, 27(8):861-874.

35. Menard SW: Applied logistic regression analysis. Thousand Oaks, CA: Sage Publications; , Second 2002.

36. Rothman KJ: No Adjustments Are Needed for Multiple Comparisons. Epidemiology 1990, 1(1):43-46.

37. Beck AT, Weishaar ME: Suicide risk assessment and prediction. Crisis 1990, 2:22-30.

38. Beck AT, Steer RA, Kovacs M, Garrison B: Hopelessness and eventual suicide: a 10-year prospective study of patients hospitalized with suicidal ideation. Am J Psychiatry 1985, 142(5):559-63.

39. Brown GK, Beck AT, Steer RA, Grisham JR: Risk factors for suicide in psychiatric outpatients: a 20-year prospective study. Journal of Consulting and Clinical Psychology 2000, 68:371-377.

40. Hawton K, Sutton L, Haw C, Sinclair J, Harriss L: Suicide and attempted suicide in bipolar disorder: a systematic review of risk factors. J Clin Psychiatry 2005, 66(6):693-704.

41. Diefenbach GJ, Woolley SB, Goethe JW: The association between selfreported anxiety symptoms and suicidality. J Nerv Ment Dis 2009, 197(2):92-7.

42. Cougle JR, Keough ME, Riccardi CJ, Sachs-Ericsson N: Anxiety disorders and suicidality in the National Comorbidity Survey-Replication. J Psychiatr Res 2009, 43(9):825-9.

43. Morrison $\mathrm{R}, \mathrm{O}^{\prime}$ Connor $\mathrm{RC}$ : The role of rumination, attentional biases and stress in psychological distress. Br J Psychol 2008, 99(2):191-209, [Epub 2007 Jun 6]

44. Rudd MD, Dahm PF, Rajab MH: Diagnostic comorbidity in persons with suicidal ideation and behavior. Am J Psychiatry 1993, 150(6):928-34.

45. Nordentoft M: Prevention of suicide and attempted suicide in Denmark. Epidemiological studies of suicide and intervention studies in selected risk groups. Dan Med Bull 2007, 54(4):306-69.

46. Starcevic V, Bogojevic G, Marinkovic J, Kelin K: Axis I and axis II comorbidity in panic/agoraphobic patients with and without suicidal ideation. Psychiatry Res 1999, 88(2):153-61.

47. Isometsä ET, Henriksson MM, Heikkinen ME, Aro HM, Marttunen MJ, Kuoppasalmi Kl, Lönnqvist JK: Suicide among subjects with personality disorders. Am J Psychiatry 1996, 153(5):667-73.

48. Escobar Jl, Gureje O: Influence of cultural and social factors on the epidemiology of idiopathic somatic complaints and syndromes. Psychosom Med 2007, 69(9):841-5.

49. Ebert D, Martus P: Somatization as a core symptom of melancholic type depression. Evidence from a cross-cultural study. J Affect Disord 1994, 32(4):253-6.

\section{Pre-publication history}

The pre-publication history for this paper can be accessed here: http://www.biomedcentral.com/1471-244X/10/110/prepub

doi:10.1186/1471-244X-10-110

Cite this article as: Yaseen et al: Construct development: The Suicide Trigger Scale (STS-2), a measure of a hypothesized suicide trigger state. BMC Psychiatry 2010 10:110. 